\title{
Reentrant and whirling hexagons in non-Boussinesq convection
}

\author{
S. Madruga ${ }^{1}$ and H. Riecke ${ }^{2}$ \\ 1 Max-Planck-Institute for Physics of Complex Systems, 01187 Dresden, Germany \\ 2 Department of Engineering Sciences and Applied Mathematics, Northwestern University, \\ Evanston, IL 60208, USA
}

\begin{abstract}
We review recent computational results for hexagon patterns in nonBoussinesq convection. For sufficiently strong dependence of the fluid parameters on the temperature we find reentrance of steady hexagons, i.e. while near onset the hexagon patterns become unstable to rolls as usually, they become again stable in the strongly nonlinear regime. If the convection apparatus is rotated about a vertical axis the transition from hexagons to rolls is replaced by a Hopf bifurcation to whirling hexagons. For weak non-Boussinesq effects they display defect chaos of the type described by the two-dimensional (2D) complex Ginzburg-Landau equation. For stronger non-Boussinesq effects the Hopf bifurcation becomes subcritical and localized bursting of the whirling amplitude is found. In this regime the coupling of the whirling amplitude to (small) deformations of the hexagon lattice becomes important. For yet stronger non-Boussinesq effects this coupling breaks up the hexagon lattice and strongly disordered states characterized by whirling and lattice defects are obtained.
\end{abstract}

\section{Introduction}

Rayleigh-Bénard convection has been a paradigmatic system for studies of spontaneously forming spatial or spatio-temporal patterns (e.g. [1]) far from equilibrium. In recent years exciting results have been obtained for the stability and dynamics of structures that are connected with roll convection. In particular, the spiral-defect chaos obtained in convection of fluids with low Prandtl number [2-7] and domain chaos [8-11] driven by the Küppers-Lortz instability [12] in rotating systems have attracted notable interest. In most of these investigations the systems have been kept close to the Oberbeck-Boussinesq approximation by minimizing the dependence of the fluid parameters on the temperature to avoid the appearance of cellular or hexagonal structures.

Variations of the fluid parameters with the temperature, i.e. non-Oberbeck-Boussinesq effects, break the up-down symmetry and give rise to hexagons at the onset of the convection. Weakly nonlinear analysis shows that typically hexagons become unstable to rolls further above threshold [13]. This scenario has been confirmed in quite a number of experimental investigations [1] and it has often been assumed that in convection hexagonal patterns are confined to the regime close to onset. Presumably due to this assumption, hexagonal convection in strongly nonlinear non-Boussinesq convection has not received as much attention.

Recently a couple of experiments $[14,15]$ showed that even in the strongly nonlinear regime hexagonal convection patterns can be observed stably. In one case hexagons were observed at relatively high Rayleigh numbers $\left(\epsilon \equiv\left(R-R_{c}\right) / R_{c} \approx 3.5\right)$ under conditions in which the Oberbeck-Boussinesq approximation was quite well satisfied. Correspondingly, hexagons with up- and with down-flow in the center were observed to coexist in adjacent domains [14]. 
Subsequently, the linear stability of up- and down-hexagons in this regime of Boussinesqconvection was confirmed by a numerical stability analysis $[16,17]$. In the other case a strongly non-Boussinesq situation was investigated and hexagons were observed not only close to threshold but also again as reentrant hexagons at higher Rayleigh numbers, $\epsilon=\mathcal{O}(1)[15]$. As the non-Boussinesq effects were increased the intermediate $\epsilon$-range over which rolls were the preferred planform shrank and eventually hexagons were found to dominate rolls from directly at onset all the way to $\epsilon=\mathcal{O}(1)$. The restabilization of hexagons at larger $\epsilon$ was attributed to the high compressibility of the fluid; the experiment was performed with $\mathrm{SF}_{6}$ near its thermodynamical critical point [15].

If the chiral symmetry of the convective apparatus is broken by rotating it about the vertical axis the Küppers-Lortz instability [12] leads to new complex dynamics. Above a critical frequency rate domain chaos sets in, which is characterized by a persistent switching between roll patches with different orientation. In the non-Boussinesq case hexagons are steady near threshold. However weakly non-linear theory predicts a secondary Hopf bifurcation to oscillations in which the three modes forming the hexagonal lattice oscillate with the same frequency but with a phase shift of $2 \pi / 3[18,19]$.

Here we review recent computational results for strongly nonlinear non-Boussinesq convection [20-22] and illustrate them further with additional data. The paper is organized as follows. After outlining the basic equations for non-Boussinesq convection (section 2) we discuss reentrant hexagons in fluids with high and to some extent also with low Prandtl number (section 3). The reentrance is clearly illustrated with numerical simulations in relatively large systems. In section 4 we address non-Boussinesq convection in rotating systems. We review the transition to whirling hexagons in the super- and the subcritical case, present new results for a period-doubled state, and illustrate the mechanism underlying the apparently persistent whirling chaos, which involves defects in the hexagon lattice. Conclusions are given in section 5.

\section{Basic equations}

The basic equations that we use for the description of non-Boussinesq convection have been discussed in detail previously $[21,23]$. We therefore sketch here only a brief summary. We consider a horizontal fluid layer of thickness $h$, density $\rho$, kinematic viscosity $\nu$, heat conductivity $\lambda$, thermal diffusivity $\kappa$, and specific heat $c_{p}$. The system is heated from below (at temperature $T_{1}$ ) and cooled from above (at temperature $T_{2}<T_{1}$ ), and in the rotating case it rotates about a vertical axis with angular velocity $\omega$, otherwise $\omega=0$.

To render the governing equations and boundary conditions dimensionless we choose the length $h$, the time $h^{2} / \kappa_{0}$, the velocity $\kappa_{0} / d$, the pressure $\rho_{0} \nu_{0} \kappa_{0} / h^{2}$, and the temperature $T_{s}=\nu_{0} \kappa_{0} / \alpha_{0} g h^{3}$ as the respective scales. The subscripted quantities refer to the respective values at half depth of the fluid layer in the conductive state. The non-dimensionalization gives rise to three dimensionless quantities: the Prandtl number $\operatorname{Pr}=\nu_{0} / \kappa_{0}$, the Rayleigh number $R=\alpha_{0} \Delta T g h^{3} / \nu_{0} \kappa_{0}$, and the dimensionless rotation rate $\Omega=\omega h^{2} / \nu_{0}$. Furthermore, we write the equations in terms of the dimensionless momentum density $v_{i}=\rho h u_{i} / \rho_{0} \kappa_{0}$ instead of the velocities $u_{i}$. The dimensionless form of the temperature $\hat{T}=T / T_{s}$, heat conductivity $\hat{\lambda}=\lambda / \lambda_{0}$, density $\hat{\rho}=\rho / \rho_{0}$, kinematic viscosity $\hat{\nu}=\nu / \nu_{0}$, and specific heat $\hat{c}_{p}=c_{p} / c_{p 0}$ will be used in the ensuing equations and the hats dropped for simplicity. In dimensionless form the equations for the momentum, mass conservation and heat are then given, respectively, by

$$
\begin{aligned}
& \frac{1}{P r}\left(\partial_{t} v_{i}+v_{j} \partial_{j}\left(\frac{v_{i}}{\rho}\right)\right)=-\partial_{i} p+\delta_{i 3}\left(1+\gamma_{1}\left(-2 z+\frac{\Theta}{R}\right)\right) \Theta \\
&+\partial_{j}\left[\nu \rho\left(\partial_{i}\left(\frac{v_{j}}{\rho}\right)+\partial_{j}\left(\frac{v_{i}}{\rho}\right)\right)\right]+2 \Omega \epsilon_{i j 3} v_{j} \\
& \partial_{j} v_{j}=0, \\
& \partial_{t} \Theta+\frac{v_{j}}{\rho} \partial_{j} \Theta=\frac{1}{\rho c_{p}} \partial_{j}\left(\lambda \partial_{j} \Theta\right)-\gamma_{3} \partial_{z} \Theta-R \frac{v_{z}}{\rho}\left(1+\gamma_{3} z\right) .
\end{aligned}
$$


with the dimensionless boundary conditions

$$
\mathbf{v}(x, y, z, t)=\Theta(x, y, z, t)=0 \quad \text { at } \quad z= \pm \frac{1}{2} .
$$

Here $\Theta$ is the deviation of the temperature field from the basic conductive profile, $\delta_{i j}$ is the Kronecker delta, and $\epsilon_{i j 3}$ the Levi-Civita tensor. Summation over repeated indices is assumed.

In the rotating case, we assume small rotation rates and neglect the centrifugal force term relative to gravity, i.e., we assume $\Omega^{2} r \ll g$. Notice that rotation breaks the chiral symmetry through the presence of the Coriolis term $2 \boldsymbol{\Omega} \times \boldsymbol{u}$.

We consider the non-Boussinesq effects to be weak and retain in a Taylor expansion of all material properties only the leading-order temperature dependence beyond the OB approximation. For the density this implies also a quadratic term with coefficient $\gamma_{1}$. It contributes, however, only to the buoyancy term in (1); in all other expressions it would constitute only a quadratic correction to the leading-order non-Boussinesq effect. Thus, the remaining temperature dependence of the fluid parameters $\rho, \nu, \lambda$, and $c_{p}$ in $(1,2,3)$ is taken to be linear

$$
\begin{aligned}
& \rho(\Theta)=1-\gamma_{0}\left(-z+\frac{\Theta}{R}\right), \\
& \nu(\Theta)=1+\gamma_{2}\left(-z+\frac{\Theta}{R}\right), \\
& \lambda(\Theta)=1+\gamma_{3}\left(-z+\frac{\Theta}{R}\right), \\
& c_{p}(\Theta)=1+\gamma_{4}\left(-z+\frac{\Theta}{R}\right) .
\end{aligned}
$$

The coefficients $\gamma_{i}$ give the difference of the respective fluid properties across the layer. They depend therefore linearly on the Rayleigh number,

$$
\gamma_{i}(\Delta T)=\gamma_{i}^{c}\left(\frac{R}{R_{c}}\right)=\gamma_{i}^{c}(1+\epsilon)
$$

where $\gamma_{i}^{c}$ is the value of $\gamma_{i}$ at the onset of convection and $\epsilon \equiv\left(R-R_{c}\left(\gamma_{i}^{c}\right)\right) / R_{c}\left(\gamma_{i}^{c}\right)$ is the reduced Rayleigh number.

The linear analysis yields the critical Rayleigh number $R_{c}$ as well as the critical wavenumber $q_{c}$. Both depend on the non-Boussinesq-coefficients $\gamma_{i}^{c}$ which in turn depend on $R_{c}$. Thus, in principle one obtains an implicit equation for the $\gamma_{i}^{c}$. The shift in the critical Rayleigh number away from the classical value $R_{c}=1708$ due to the non-Boussinesq-effects is, however, quite small (less than 1 percent) and therefore the resulting change in the $\gamma_{i}^{c}$ is negligible. In this paper we therefore choose the $\gamma_{i}^{c}$ corresponding to $R_{c}=1708$.

Details of the numerical scheme used to determine the stability of patterns in the strongly non-linear regime, and for the integration of the Navier-Stokes equations can be found in [21].

\section{Reentrant hexagons}

The classic weakly nonlinear analysis for weakly non-Boussinesq convection [13] predicts that not far above threshold the hexagons become unstable to a mixed-mode, which in turn is also unstable, resulting in a transition to roll convection. This transition has been addressed in experimental studies in $\mathrm{CO}_{2}[24]$ and water $[25,26]$, respectively. In both studies a transition to rolls was observed. The quantitative agreement with the weakly nonlinear theory was, however, limited. Among the reasons identified for the poor agreement were the boundaries, which enhance the tendency towards rolls. 


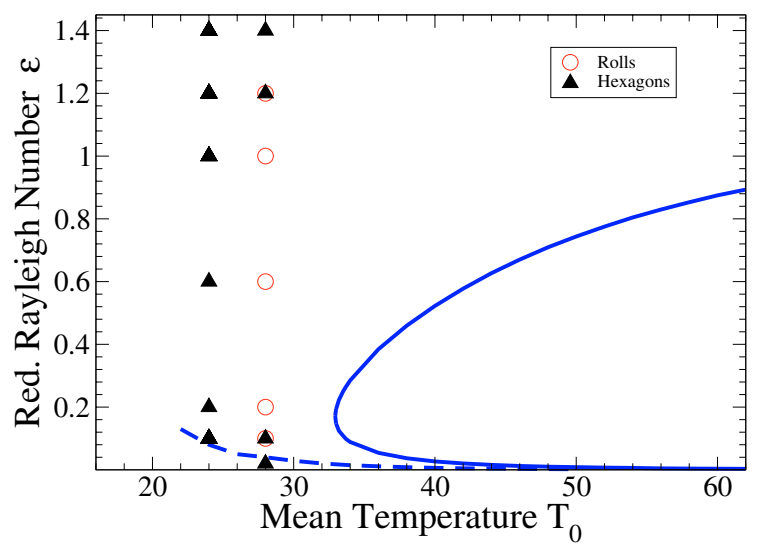

Fig. 1. (Color online). Phase diagram showing the results of simulations for a layer of water with thickness $h=1.8 \mathrm{~mm}$ in a circular cell with diameter $L=16 \times 2 \pi / q_{c}$. The simulations have been carried out for $T_{0}=24^{\circ} \mathrm{C}$ and $28^{\circ} \mathrm{C}$ (for further parameters see figures 2 and 3 ). Circles correspond to rolls and triangles to hexagons. The full and dashed line correspond to the stability limits for amplitude instabilities of hexagons and rolls, respectively, as determined by the Galerkin calculation.

In recent work on the stability of fully nonlinear hexagon solution of the Navier-Stokes equations we have again addressed the transition from hexagons to rolls [20,21] and found that a further cause for disagreement between weakly nonlinear theory and experiments may be given by the fact that even for seemingly moderate non-Boussinesq effects the transition from hexagons to rolls can be shifted towards larger values of $\epsilon$ and can, in fact, disappear altogether.

Results of our stability calculation for a layer of water of height $h=1.8 \mathrm{~mm}$ are shown as a function of the mean temperature $T_{0}$ of the layer in figure 1 . The mean temperature is defined as $T_{0}=\left(T_{1}+T_{2}\right) / 2$, where $T_{1}$ and $T_{2}$ are the temperatures at the bottom and top plates respectively, and $T_{0}$ is kept constant in the simulations of the Navier-Stokes and energy equations. Hexagons are stable below and to the left of the solid line, whereas rolls are linearly stable to hexagons above the dashed line. With decreasing mean temperature (and with decreasing layer height) the critical temperature for the onset of convection increases and with it the variation of the fluid parameters across the layer. In addition, for lower mean temperatures the temperature dependence of the density of water develops a strong quadratic contribution. Thus, with decreasing mean temperature the non-Boussinesq effects increase. This shifts the line indicating the instability of hexagons to rolls to larger values of $\epsilon$. More importantly yet, we found that the hexagons can regain stability with respect to rolls if the Rayleigh number is increased well beyond the weakly nonlinear regime. This line of reentrance is shifted towards lower value of $\epsilon$ as the non-Boussinesq effects become more pronounced and in fact merges eventually with the lower stability limit of the hexagons at a temperature $T_{m}$. Below $T_{m}$ the hexagons do not undergo any instability with respect to rolls over the whole range of $\epsilon$ shown.

More detailed stability analyses of the hexagons with periodic boundary conditions using a general Galerkin-Floquet ansatz to capture side-band instabilities show that the only relevant side-band perturbations are long-wave and steady, as is the case in the weakly nonlinear regime. The range of stable wavenumbers $q$ shifts towards smaller values of $q$ as $\epsilon$ is increased. This reflects the fact that even in the absence of non-Boussinesq effects hexagons can become linearly stable relatively far from threshold, $\epsilon>2$. These hexagons are, however, stable with respect to side-band instabilities only for relatively low values of $q[16]$.

A seemingly similar reentrance of hexagon convection has been observed by Roy and Steinberg in $\mathrm{SF}_{6}$ near the thermodynamical critical point [15]. There it was argued that since the non-Boussinesq effects in that system are not very large near onset the reentrance is due to the large compressibility of the fluid in this parameter regime. By assuming that the working fluid is incompressible, which is an excellent approximation for water, our computations show that high compressibility is not needed for reentrance. The significance of the compressibility for the occurrence of the reentrance has also been called into question in $[27,28]$. Moreover, 


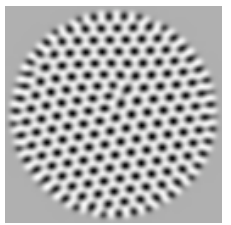

$\varepsilon=0.1$

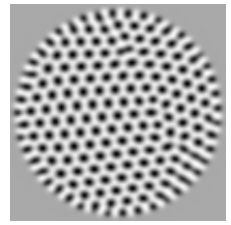

$\varepsilon=0.2$

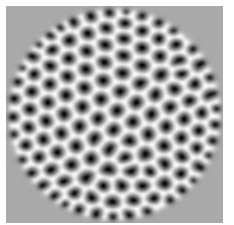

$\varepsilon=1.4$

Fig. 2. Series of snapshots in a circular cell of water of thickness $h=1.8 \mathrm{~mm}$ and mean temperature $T_{0}=24^{\circ} \mathrm{C}$. The diameter of the cell is $L=16 \times 2 \pi / q_{c}$, and the snapshots correspond to an integration time of $600 t_{v}$. The corresponding non-Boussinesq coefficients are $\gamma_{0}^{c}=0.0039, \gamma_{1}^{c}=0.3326, \gamma_{2}^{c}=$ $-0.3641, \gamma_{3}^{c}=0.0467, \gamma_{4}^{c}=-0.0026$, and the Prandtl number $\operatorname{Pr}=6.3$.

the wavenumber of the reentrant hexagons of [15] does not decrease with increasing $\epsilon$, but stays quite close to $q_{c}$, whereas a characteristic feature of the reentrant hexagons found in our computation is that the wavenumber is significantly lower than $q_{c}$ (cf. [16]).

With respect to the experiments in water [25] our stability computations show that even in that system reentrance should have been accessible just slightly above the Rayleigh numbers investigated in those experiments $(0<\epsilon<0.14)$. For the experiment in $\mathrm{CO}_{2}[24]$ we find that the parameters correspond to the regime in which hexagons do not become unstable to rolls at all up to $\epsilon=1$, similar to the situation shown in figure 1 for water for $T_{0}<T_{m}$.

To address the influence of the side walls we have performed numerical simulations of the Navier-Stokes equations in which we mimic the circular sidewalls by applying a strong radial subcritical ramp in the Rayleigh number that suppress any convection outside a certain radius [3]. The system size is chosen to be twice as large as in [20], i.e. here $L=16 \times 2 \pi / q_{c}$. Random initial conditions are used. Figure 2 shows snapshots of simulations for a mean temperature of $T_{0}=24^{\circ} \mathrm{C}$. Consistent with the phase diagram shown in figure 1 no transition from hexagons to rolls is found. As expected from the shift of the stable wavenumber band towards smaller value of $q$ the hexagon wavenumber is decreasing with increasing $\epsilon$. Note that the defects that cause the change in wavenumber do not trigger a transition to rolls.

For larger mean temperature, $T_{0}=28^{\circ} \mathrm{C}$, the non-Boussinesq effects are weaker. As a consequence, for intermediate values of $\epsilon$ rolls invade the hexagon pattern from the side walls similar to the experiments of $[24,25]$ and for values of $\epsilon$ in the range $0.6<\epsilon<0.1$ disordered roll patterns are obtained, which are characteristically perpendicular to the sidewalls (figure 3 ). For $\epsilon=1.2$ the hexagons take over again and we find a new interesting scenario: coexistence between rolls and reentrant hexagons. This state was not obtained in our previous simulations of water due to the small aspect ratio used [21] nor in simulations of reaction diffusion system [29]. At $\epsilon=1.4$ the non-Boussinesq effects become strong enough to remove all the rolls and the reentrant hexagons take up the whole convective cell.

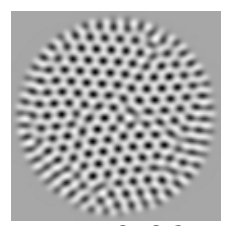

$\varepsilon=0.02$

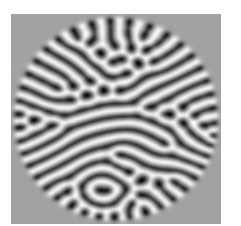

$\varepsilon=1.0$

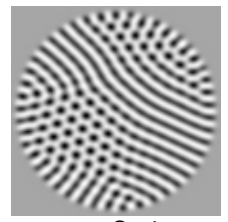

$\varepsilon=0.1$

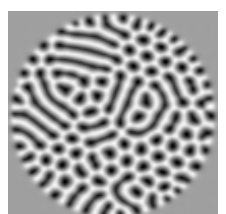

$\varepsilon=1.2$

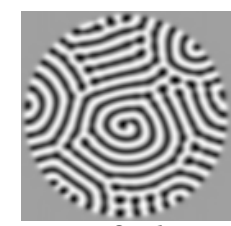

$\varepsilon=0.6$

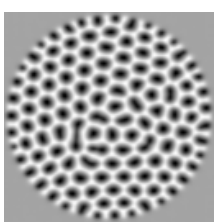

$\varepsilon=1.4$

Fig. 3. Sequence of snapshots for $T_{0}=28^{\circ} \mathrm{C}$ in a circular cell of water of thickness $h=1.8 \mathrm{~mm}$. The diameter of the cell is $L=16 \times 2 \pi / q_{c}$, and the snapshots correspond to an integration time of $300 t_{v}$. The corresponding non-Boussinesq coefficients are $\gamma_{0}^{c}=0.0036, \gamma_{1}^{c}=0.2122, \gamma_{2}^{c}=-0.2725$, $\gamma_{3}^{c}=0.0352, \gamma_{4}^{c}=-0.0013$, and the Prandtl number $\operatorname{Pr}=5.7$. 
The results of the simulations are summarized in figure 1 with triangles denoting hexagons and circles marking roll states. Compared to figure 9 in [21] the regime of disordered roll patterns is shifted towards larger $T_{0}$, i.e towards weaker non-Boussinesq effects. This is due to the larger convection cell used in the present simulations. This reduces the influence of the sidewalls. They enhance the nucleation of rolls, which then can invade the hexagonal pattern in the bulk.

\section{Convection with rotation}

If the whole system is rotated about a vertical axis the Coriolis force breaks the chiral symmetry, which transforms the steady bifurcation off the hexagons to the unstable mixed mode into a Hopf bifurcation to whirling hexagons [18,19]. In [30,31] the complex Ginzburg-Landau equation describing spatial modulations of the complex oscillation amplitude $\mathcal{H}$ of the whirling hexagons has been derived from three coupled Ginzburg-Landau equations that provide the weakly nonlinear description of the hexagons,

$$
\partial_{T} \mathcal{H}=a \mathcal{H}+d \nabla^{2} \mathcal{H}-c \mathcal{H}|\mathcal{H}|^{2} .
$$

In principle, this CGL is still coupled to the phase modes of the underlying hexagon pattern. However, at the band center $q_{c}$ the coupling coefficients vanish, irrespective of the coefficients of the three coupled Ginzburg-Landau equations. Moreover, the imaginary parts of the coefficients $d$ and $c$ are always in the regime of bistability of the homogeneous oscillations and defect chaos $[32]$.

Recently, we have investigated this Hopf bifurcation within the fully nonlinear Navier-Stokes equations (1)-(3) and have in particular obtained the coefficients of the CGL (10) for realistic fluid parameters [22]. To this end we have performed a numerical linear stability analysis of the steady hexagons employing a Galerkin expansion of the velocity and temperature fields for the Floquet analysis.

The linear coefficient $a \equiv a_{r}+i a_{i}$ is readily obtained from the growth rate and frequency of the perturbation associated with the Hopf bifurcation. The calculation of the diffusion and dispersion coefficient $d \equiv d_{r}+i d_{i}$ is a bit more involved. It captures the dependence of the complex growth rate $\sigma$ of the oscillatory Hopf mode on slow spatial modulations of its amplitude, $\sigma=-s^{2} d$, where $s$ is the wavenumber of the modulation, i.e. the Floquet exponent. Note, that the dependence of the growth rate of the translation mode (rather than the Hopf mode) on the Floquet parameter would yield the phase diffusion coefficients. We choose a discrete number of modulation wavevectors $\mathbf{s}$ typically in the range $0.01 \leq|\mathbf{s}| / q_{c} \leq 1$ for the computations and obtain $d$ by a fit of $\sigma$ to the equation $\sigma=-s^{2} d$. Figure 4 shows the ratio $d_{i} / d_{r}$ for $T_{0}=14^{\circ} \mathrm{C}$ and $h=4.92 \mathrm{~mm}$ as a function of the deviation of wavenumber of the underlying hexagons from the critical wavenumber, $\Delta q \equiv q-q_{c}$. Within the coupled Ginzburg-Landau equations $d_{i} / d_{r}$ vanishes at the bandcenter, $\Delta q=0$, and is quadratic in $\Delta q$. The fully nonlinear computations of the Navier-Stokes equations confirm this behavior quite well.

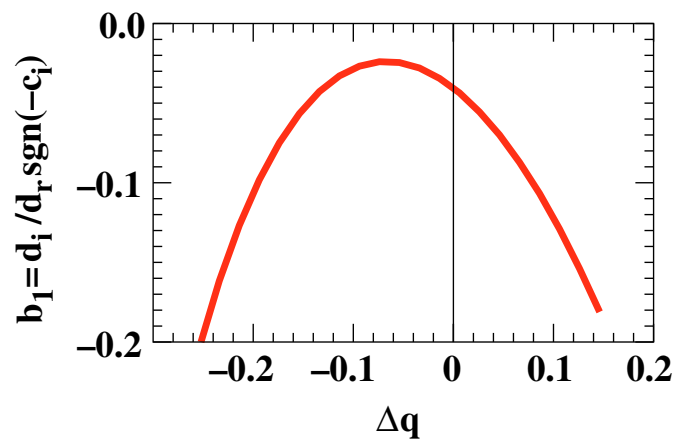

Fig. 4. (Color online). Dependence of the ratio $d_{i} / d_{r}$ on the wavenumber $\Delta q \equiv q-q_{c} . T_{0}=14^{\circ} \mathrm{C}$, $\Omega=65$, non-Boussinesq coefficients $\gamma_{0}^{c}=0.0009$, $\gamma_{1}^{c}=0.2937, \gamma_{2}^{c}=-0.1681, \gamma_{3}^{c}=0.0215, \gamma_{4}^{c}=$ -0.0022 , and Prandtl number $\operatorname{Pr}=8.2$. 
For the calculation of the non-linear coefficient $c \equiv c_{r}+i c_{i}$ we perform direct simulations of the Navier-Stokes equations (1-4) in a small box of size $L=2 \times 2 \pi / q_{c}$ to avoid side-band perturbations and spatial modulations of the oscillation amplitude. Therefore, from the real part $\mathrm{d} H / \mathrm{d} t=a_{r}|\mathcal{H}|+c_{r}|\mathcal{H}|^{3}$ we extract $c_{r}$ by fitting the time derivative of the amplitude of the Hopf mode as a function of its amplitude (notice that we extracted $a_{r}$ in the previous step through a stability linear analysis). Finally, from the imaginary part $\omega=a_{i}+c_{i}|\mathcal{H}|^{2}$ we obtain $c_{i}$ by fitting the oscillatory frequency of the Hopf mode as a function of its amplitude.

For $T_{0}=14^{\circ} \mathrm{C}$ and $h=4.92 \mathrm{~mm}$ with $\Omega=65$ we obtain coefficients that match closely the expectations based on the weakly nonlinear theory provided by the three coupled GinzburgLandau equations. In particular, we confirm that the system should exhibit bistability between homogeneous oscillations and defect chaos. Direct simulations in sufficiently large system confirmed this prediction. When starting with ordered hexagons and small perturbations that break the chiral symmetry, i.e. the three dominant wavevectors making up the hexagon pattern are given slightly different amplitudes, we obtain stable homogeneous oscillations, i.e. all hexagons whirl in phase with each other. To obtain the defect-chaos, defects need to be introduced into the complex oscillation amplitude. Note, however, that preferably there should be no defects in the underlying hexagon pattern. We therefore start with initial conditions in which the complex amplitude exhibits phase winding and its phase changes by $2 \pi$ across the system. In our simulations we find that this phase-modulated state is linearly unstable to side-band perturbations of the complex oscillation amplitudes which lead to defects in that amplitude. Through their wavenumber selection the defects induce the creation of further defects, without any significant deformations of the hexagon lattice.

For somewhat stronger non-Boussinesq effects $\left(T_{0}=12^{\circ} \mathrm{C}\right.$ with $\left.4.92 \mathrm{~mm}\right)$ we find that the Hopf bifurcation becomes subcritical. Since within the three coupled Ginzburg-Landau equations the Hopf bifurcation is always supercritical, this shows that the Hopf bifurcation occurs now beyond their regime of validity. Nevertheless, near the parameter values for which the bifurcation changes direction the whirling hexagons can still be described by a complex Ginzburg-Landau equation if a quintic saturating term $-g \mid \mathcal{H}^{4} \mathcal{H}$ is retained. Following the same procedure as before we determine the coefficients of this quintic equation and obtain $d=22.4+0.389 i, c=-12.96+84.7 i, g=42.53+17.20 i$. The dependence of $d_{i} / d_{r}$ on the hexagon wavenumber is shown in figure 5 . In contrast to the case $T_{0}=14^{\circ} \mathrm{C}$ shown in figure 4 , the dependence is now essentially linear near $q_{c}$, reflecting again the fact that the Hopf bifurcation occurs at larger amplitudes for which the coupled Ginzburg-Landau equations are no longer valid. The inset of figure 5 shows that the dependence of $\omega$ with respect to $\mathcal{H}^{2}$ is almost linear, and thus the quintic contribution $g_{i}|\mathcal{H}|^{4}$ to the frequency is very small in this regime.

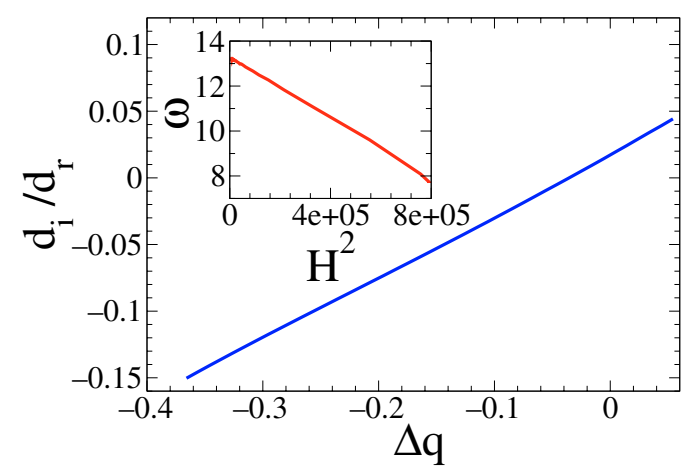

Fig. 5. Dependence of the ratio $d_{i} / d_{r}$ on the wavenumber $\Delta q \equiv q-q_{c}$ (main figure), and dependence of the frequency of the Hopf mode on its amplitude (inset). The ratio $d_{i} / d_{r}$ is extracted from linear stability calculations and the frequency from numerical simulations in a small box of size $L=2 \times 2 \pi / q_{c}$, with $T_{0}=12^{\circ} \mathrm{C}, \Omega=65$, non-Boussinesq coefficients $\gamma_{0}^{c}=0.00100, \gamma_{1}^{c}=0.4885, \gamma_{2}^{c}=-0.2281$, $\gamma_{3}^{c}=0.0287, \gamma_{4}^{c}=-0.00320$, and Prandtl number $\operatorname{Pr}=8.7$. 


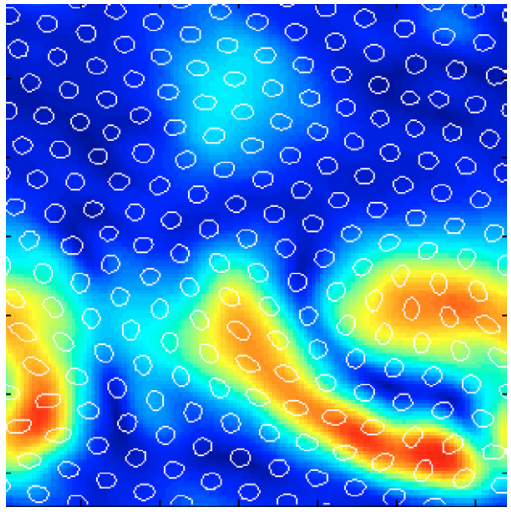

Fig. 6. (Color online). Localized bursting of the oscillation amplitude. Color gives magnitude of oscillation amplitude (red: maximal, blue: zero). White lines indicate underlying hexagon pattern and show the deformation of the hexagon cells by the whirling.

Direct simulations of the Navier-Stokes equations reveal that in this regime typical states exhibit localized unsteady bursts in the oscillation amplitude as shown in figure 6 . The white lines give the hexagonal cells, showing the deformations due to the whirling, and the magnitude $|\mathcal{H}|$ of the oscillation amplitude is shown in the color scale (red: maximal, blue: zero). We extract the complex oscillation amplitude $\mathcal{H}$ from the simulation by demodulation with respect to the wavenumber of the hexagons and the dominant frequency of the oscillation. To do so, we first evaluate the Fourier transform of the time series given by the amplitude at each point of the numerical grid, and then shift the resulting temporal Fourier spectrum by the Hopf frequency. In this way we capture the slow time evolution. To obtain the slow spatial modulations, we compute the spatial Fourier transform of the shifted temporal Fourier transform, shift it such that one of the six dominant peaks making up the hexagonal lattice is moved to the origin, and remove the spectral content beyond a radius of $\sqrt{3} q_{c} / 2$.

To assess the validity of the quintic complex Ginzburg-Landau equation we simulate it numerically using the parameters obtained from the simulations of the Navier-Stokes equation. We find that using the same system size the quintic CGL does not exhibit the bursting found in the Navier-Stokes simulations since the homogeneous oscillations become unstable to sideband perturbations only for smaller wavenumbers. If, however, the system size is chosen larger, similar bursting is obtained, as shown in figure 7. A more careful analysis shows that in this regime the coupling to the phase equations for the deformations of the underlying hexagons cannot be ignored [22]. These deformations represent changes in the local wavenumber of the hexagons. As our numerical stability analysis of the steady hexagons shows, in this regime the growth rate of the Hopf mode increases significantly with decreasing hexagon wavenumber. Thus, due to the deformation of the hexagons by the whirling the local growth rate within a burst is enhanced and allows this burst to persist.

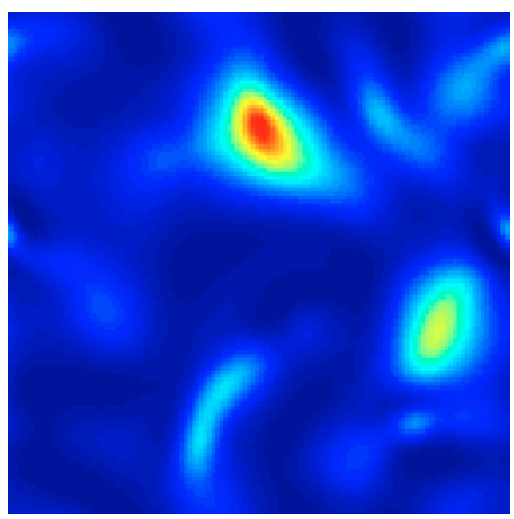

Fig. 7. (Color online). Localized bursting of the oscillation amplitude in the quintic complex Ginzburg-Landau equation. Parameters correspond to those of figure 6 except for a larger system size. 

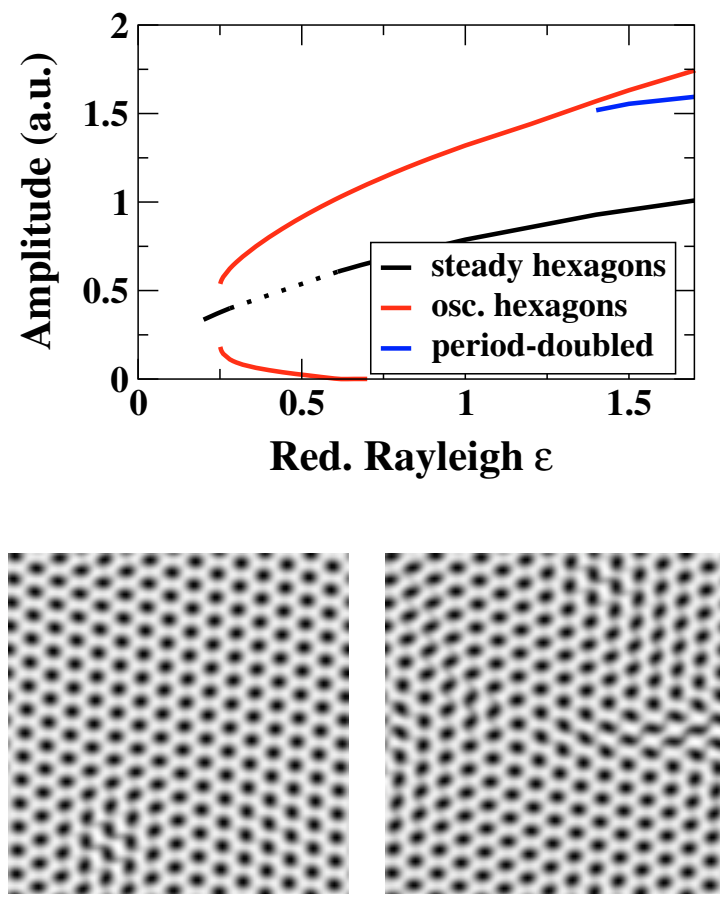

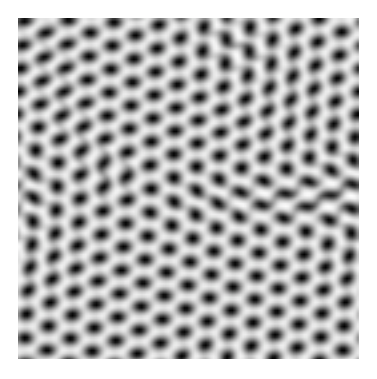

Fig. 8. (Color online). Bifurcation diagram of non-Boussinesq convection with rotation for the subcritical case with $T_{0}=12^{\circ} \mathrm{C}$ and $\Omega=65$. Blue line corresponds to the minimal oscillation amplitude of the period-doubled orbit, the maximal oscillation amplitude is marked by the red line.

Fig. 9. Bursting state in water for $T_{0}=12^{\circ} \mathrm{C}$, $\Omega=65$ and $\epsilon=0.7$. The snapshots come from simulations of equations (1-4) in a cell of size $L=16 \times 2 \pi / q_{c}$ using random initial conditions. Left snapshot corresponds to time $t=663 t_{v}$ and right $t=699 t_{v}$.

Increasing the Rayleigh number, the steady hexagons again become stable, as in the nonrotating case discussed above. The corresponding bifurcation diagram is shown in figure 8 . Nevertheless, for suitable initial conditions we still find irregular bursting of the whirling activity for $\epsilon=0.7$, but not for $\epsilon=0.9$. Two snapshots depicting whirling localized within domains of varying sizes are shown in figure 9. Compared to the bursting obtained for $\epsilon=0.5[22]$, the fluctuations in the whirling activity, $I(t)=N^{-1} \int_{|\mathcal{H}|>0.5|\mathcal{H}|_{\max }}|\mathcal{H}| \mathrm{d} x \mathrm{~d} y$ with $N$ denoting the temporal mean of the integral, are much smaller (figure 10). Nevertheless, persistently shifting domains of whirling hexagons are still apparent in figure 9.

Increasing $\epsilon$ further, we find a period-doubling bifurcation at $\epsilon \simeq 1.3$ as shown in figure 8 , which gives the larger (red) and smaller (blue) peak amplitude of the oscillations. Figure 11 presents a time series of the amplitude of one of the Fourier modes that forms the hexagonal lattice for $\epsilon=1.5$. The time series is obtained from the simulation of equations (1)-(4) in a small cell of size $L=2 \times 2 \pi / q_{c}$. Whether there are any further period doubling events for larger $\epsilon$ remains to be investigated. So far we have obtained this state only in simulations of small systems in which side-band perturbations of the oscillation mode as well as the hexagon pattern are negligible.

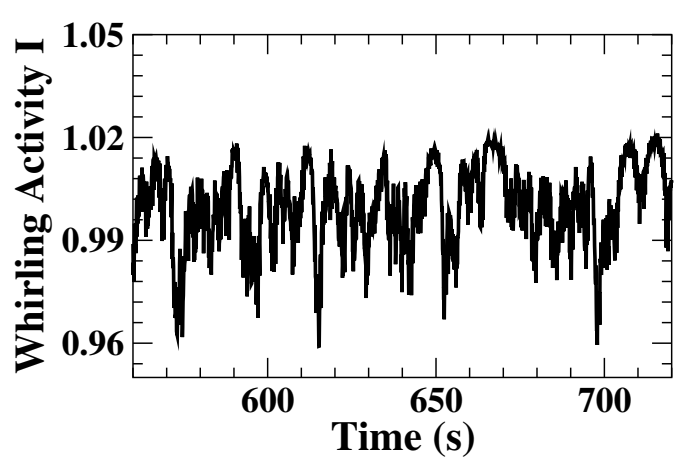

Fig. 10. Temporal evolution of the whirling activity $I(t)=N^{-1} \int_{|\mathcal{H}|>0.5|\mathcal{H}|_{\max }}|\mathcal{H}| \mathrm{d} x \mathrm{~d} y$ of the bursting state (see figure 9 ). 


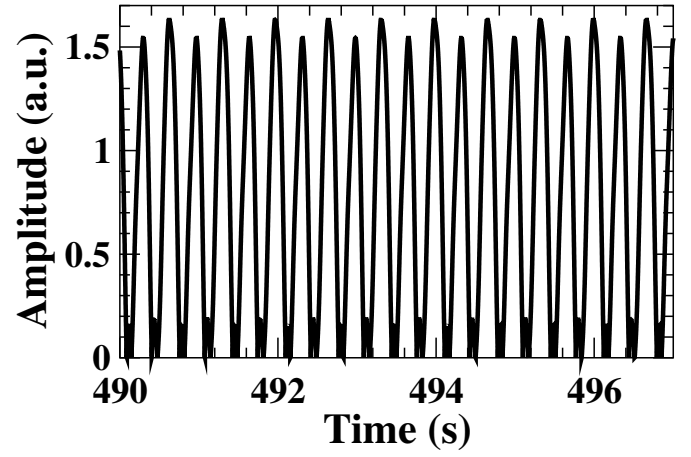

Fig. 11. Period doubling after a Hopf bifurcation in the amplitude of one of the three modes that forms a hexagonal lattice. The simulations are made in a small cell of size of $L=2 \times 2 \pi / q_{c}$ with $T_{0}=12, \epsilon=1.5$, and $\Omega=65$.

As the non-Boussinesq effects are increased yet further [23], the range over which the steady hexagons are unstable to the whirling becomes smaller and eventually the steady hexagons are linearly stable over the whole range of Rayleigh numbers considered, $0 \leq \epsilon \leq 1.2$. However, since the Hopf bifurcation is subcritical in this regime solutions with whirling hexagons still exist. In fact, the whirling activity, which does deform the underlying hexagon pattern [30,31, 33 ], is so strong that it breaks up the hexagonal lattice, introducing many defects. In weakly disordered hexagon patterns the dominant defects are typically penta-hepta defects which consist of two adjacent convection cells that have 5 and 7 immediate neighbors, respectively. The state resulting from the strong whirling activity is, however, considerably more disordered and exhibits not only such cells with coordination numbers 5 and 7 , but also a small number of cells with coordination numbers 4 and 8 , respectively [23].

For intermediate Rayleigh numbers the spatio-temporally chaotic whirling activity arises only transiently. During that transient one can qualitatively discern a mutual reinforcement of the defect formation and the whirling activity. This is illustrated in the two snapshots shown in figure 12. Whirling activity emanates from the defects in the hexagonal lattice (marked by a red and a blue circle). It in turn tends to induce other defects, see right panel. For the parameters chosen in figure 12 the whirling emanating from the defects is not strong enough and the various defects in the lattice eventually annihilate each other and the pattern becomes steady. If the mutual reinforcement is strong enough, however, persistent activity can arise. For $\epsilon=1$ the whirling invades the steady state and in systems of size $L=16 \times 2 \pi / q$ with $q=4.5$ we find that the chaotic activity persists as long as we could simulate the system (more than 300 vertical thermal diffusion times, which corresponds to more than 5 hours physical time).
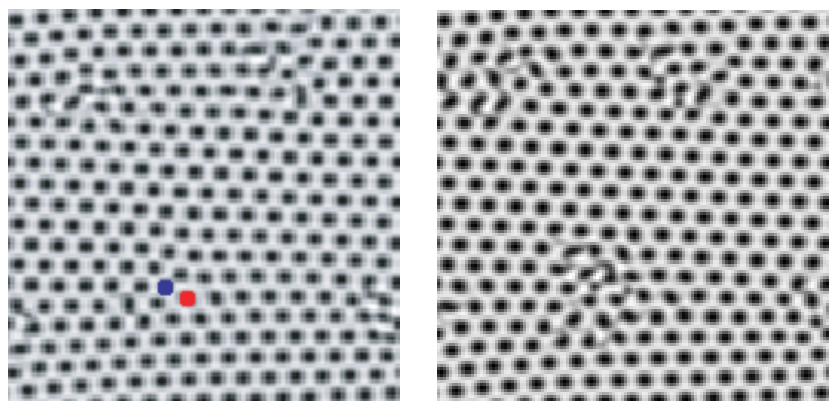

Fig. 12. (Color online). Interplay between whirling activity and defect formation. Two snapshots for $\epsilon=0.87$ and $h=4.6 \mathrm{~mm}, T_{0}=12^{\circ} \mathrm{C} \Omega=65$. Penta-hepta defects (one is marked by blue and red circle) induce whirling and whirling tends to induce defects. 


\section{Conclusion}

In this paper we have elaborated on some recent results for hexagon convection in nonBoussinesq convection [20-22]. In the non-rotating case we focused on the reentrance of hexagons as the Rayleigh number is increased. We contrasted the reentrance found in our computations for water with that found in experiments using a layer of $\mathrm{SF}_{6}$. The reentrant hexagons obtained through the mechanism elucidated with our computations generically are expected to inherit the low wavenumber that is characteristic of Boussinesq hexagons [16]. In contrast, the reentrant hexagons found in the $\mathrm{SF}_{6}$ experiments have a wavenumber close to $q_{c}$ [15], suggesting that their reentrance is due to a different mechanism.

For stronger non-Boussinesq effects the hexagons do not exhibit any linear instability to rolls (or the mixed mode) and, in fact, even defects arising through side-band instabilities of the hexagons do not necessarily lead to a transition to rolls. Instead the pattern can heal to a regular hexagon pattern with lower wavenumber. In convection in gases, which have a low Prandtl number, the side-band instabilities limiting the stable wavenumber band can be different at the low- $q$ and the high- $q$ side, which leads to transients exhibiting different degrees of disorder [20].

Similar looking reentrant hexagons have also been reported in a chemical model system, the 2D Brusselator system, for which a series of numerical simulations were performed [29]. The initial Turing instability off the homogeneous state yields hexagon patterns. With increasing control parameter these hexagons lose their stability to stripe-like patterns. For yet larger values of the control parameter the hexagons regain their stability. In contrast to the behavior found in convection in this chemical model the hexagons near onset are up-hexagons ( $\pi$-hexagons) while the reentrant hexagons are down-hexagons (0-hexagons). These results are modeled with an amplitude equation whose quadratic coefficient depends on the control parameter. Such a model has also has been used for hexagons in magnetic fluids [34] and in our previous calculations for water [21].

For rotating systems we discussed the whirling hexagons that arise from a Hopf bifurcation that takes the place of the steady bifurcation to a mixed mode in the non-rotating case. In the weakly non-Boussinesq case this transition occurs for very small convection amplitudes and the Hopf bifurcation is supercritical, as predicted by computations based on three coupled Ginzburg-Landau equations describing weakly nonlinear hexagon patterns with rotation [30]. It turns out that to leading order the oscillations can be described by the $2 \mathrm{D}$ complex Ginzburg-Landau equation. Moreover, the resulting oscillations (whirling hexagons) generically arise in the regime in which defect chaos persists despite the existence of stable plane wave and homogeneously oscillating solutions. For stronger non-Boussinesq effects the bifurcation becomes subcritical and we find localized bursting in the whirling amplitude and also whirling activity that is strong enough to tear the hexagonal lattice apart.

This paper is dedicated to the memory of Carlos Pérez-García, who was SM's thesis advisor and who introduced him to the field of pattern formation. SM is grateful for years of friendship and stimulating discussions.

We thank W. Pesch for providing us with the numerical codes used in this project and for ongoing support.

This research was supported by NSF (DMS-9804673), by the Department of Energy (DE-FG0292ER14303), and by EU under grant MRTN-CT-2004-005728 (SM).

\section{References}

1. E. Bodenschatz, W. Pesch, G. Ahlers, Ann. Rev. Fluid Mech. 32, 709 (2000)

2. S.W. Morris, E. Bodenschatz, D.S. Cannell, G. Ahlers, Phys. Rev. Lett. 71, 2026 (1993)

3. W. Decker, W. Pesch, A. Weber, Phys. Rev. Lett. 73, 648 (1994)

4. S.W. Morris, E. Bodenschatz, D.S. Cannell, G. Ahlers, Physica D 97, 164 (1996)

5. D.A. Egolf, I.V. Melnikov, W. Pesch, R.E. Ecke, Nature 404, 733 (2000) 
6. R.G. Matley, W.Y. Wong, M.S. Thurlow, P.G. Lucas, M.J. Lees, O.J. Griffiths, A.L. Woodcraft, Phys. Rev. E 63, 045301 (2001)

7. K.-H. Chiam, M.R. Paul, M.C. Cross, H.S. Greenside, Phys. Rev. E 67, 056206 (2003)

8. F. Zhong, R.E. Ecke, Chaos 2, 163 (1992)

9. E. Bodenschatz, D.S. Cannell, J.R. deBruyn, R. Ecke, Y.C. Hu, K. Lerman, G. Ahlers, Physica D 61, $77(1992)$

10. Y. Hu, R.E. Ecke, G. Ahlers, Phys. Rev. Lett. 74, 5040 (1995)

11. Y. Hu, W. Pesch, G. Ahlers, R.E. Ecke, Phys. Rev. E 58, 5821 (1998)

12. G. Küppers, D. Lortz, J. Fluid Mech. 35, 609 (1969)

13. F.H. Busse, J. Fluid Mech. 30, 625 (1967)

14. M. Assenheimer, V. Steinberg, Phys. Rev. Lett. 76, 756 (1996)

15. A. Roy, V. Steinberg, Phys. Rev. Lett. 88, 244503 (2002)

16. R.M. Clever, F.H. Busse, Phys. Rev. E 53, R2037 (1996)

17. F.H. Busse, R.M. Clever, E. Grote, Chaos Sol. Fract. 10, 753 (1999)

18. J.W. Swift, Contemporary Mathematics, vol. 28, Providence (Amer. Math. Soc., 1984), p. 435

19. A.M. Soward, Physica D 14, 227 (1985)

20. S. Madruga, H. Riecke, Phys. Rev. E (2007) (to appear)

21. S. Madruga, H. Riecke, W. Pesch, J. Fluid Mech. 548, 341 (2006)

22. S. Madruga, H. Riecke, W. Pesch, Phys. Rev. Lett. 96, 074501 (2006)

23. Y.-N. Young, H. Riecke, W. Pesch, New J. Phys. 5, 135 (2003)

24. E. Bodenschatz, J.R. deBruyn, G. Ahlers, D.S. Cannell, Phys. Rev. Lett. 67, 3078 (1991)

25. E. Pampaloni, C. Pérez-Garía, L. Albavetti, S. Ciliberto, J. Fluid Mech. 234, 393 (1992)

26. C. Pérez-García, E. Pampaloni, S. Ciliberto, Europhys. Lett. 12, 51 (1990)

27. G. Ahlers, I. Mutabazi, J.E. Wesfreid, E. Guyon (eds.), Dynamics of Spatio-Temporal Cellular Structures - Henri Bénard Centenary Review (Springer Tracts in Modern Physics, Springer, 2005)

28. J. Oh, J. Ortiz de Zárate, J.V. Sengers, G. Ahlers, Phys. Rev. E 69, 021106 (2004)

29. G. Dewel, J. Verdasca, A. de Wit, P. Borkmans, Phys. Lett. A 168, 194 (1992)

30. B. Echebarria, H. Riecke, Phys. Rev. Lett. 84, 4838 (2000)

31. B. Echebarria, H. Riecke, Physica D 143, 187 (2000)

32. H. Chaté, P. Manneville, Physica A 224, 348 (1996)

33. S. Madruga, C. Pérez-García, Int. J. Bifur. Chaos 14, 107 (2004)

34. R. Friedrichs, A. Engel, Phys. Rev. E 6402, 021406 (2001) 